\title{
Article
}

\section{Solvent Vapor Annealing-Mediated Crystallization Directs Charge Generation, Recombination and Extraction in BHJ Solar Cells}

\author{
Maxime Babics, Ru-Ze Liang, Kai Wang, Federico Cruciani, Zhipeng Kan, \\ Markus Wohlfahrt, Ming-Chun Tang, Frédéric Laquai, and Pierre M. Beaujuge \\ Chem. Mater., Just Accepted Manuscript • DOI: 10.1021/acs.chemmater.7b04286 • Publication Date (Web): 18 Dec 2017
}

Downloaded from http://pubs.acs.org on December 24, 2017

\section{Just Accepted}

"Just Accepted" manuscripts have been peer-reviewed and accepted for publication. They are posted online prior to technical editing, formatting for publication and author proofing. The American Chemical Society provides "Just Accepted" as a free service to the research community to expedite the dissemination of scientific material as soon as possible after acceptance. "Just Accepted" manuscripts appear in full in PDF format accompanied by an HTML abstract. "Just Accepted" manuscripts have been fully peer reviewed, but should not be considered the official version of record. They are accessible to all readers and citable by the Digital Object Identifier (DOI®). "Just Accepted" is an optional service offered to authors. Therefore, the "Just Accepted" Web site may not include all articles that will be published in the journal. After a manuscript is technically edited and formatted, it will be removed from the "Just Accepted" Web site and published as an ASAP article. Note that technical editing may introduce minor changes to the manuscript text and/or graphics which could affect content, and all legal disclaimers and ethical guidelines that apply to the journal pertain. ACS cannot be held responsible for errors or consequences arising from the use of information contained in these "Just Accepted" manuscripts. 


\section{Introduction}

Solution-processable bulk-heterojunction $(\mathrm{BHJ})$ solar cells relying on small-molecule (SM) donors and fullerene (e.g., $\left.\mathrm{PC}_{61} /{ }_{71} \mathrm{BM}\right)$ or "nonfullerene" acceptors are gaining attention in the field, mainly because high-efficiency figures are now in reach ${ }^{1}$ with the prospects of outperforming the more commonly studied polymer-based BHJ solar cells. ${ }^{2,3}$ Their discrete molecular structure and defined molecular weight, their synthetic accessibility and scalability, and the absence of batch-to-batch variation, are some of the main incentives for using SM donors and acceptors in BHJ solar cells. ${ }^{1,4}$ When combined with fullerene acceptors, SM donor-based $\mathrm{BHJ}$ devices can reach power conversion efficiencies (PCEs) $>11 \%,{ }^{5}$ and swapping fullerenes for "nonfullerene" acceptors yield PCEs $>10 \% .{ }^{6}$ At the origin of the gradual efficiency improvements seen in the past recent years: (i) the rationalization of material design and (ii) the systematic optimization of film processing conditions and device structure. More often than not, achieving a favorable morphology in SM donor-fullerene $\mathrm{BHJ}$ solar cells requires examining several of these parameters: solvent selection, ${ }^{7}$ donor/acceptor ratio, ${ }^{8,}, 9$ device configuration and interlayer selection, ${ }^{10,11}$ processing additives, ${ }^{9,12}$ thermal annealing, ${ }^{13}$ and more recently- post-processing "solvent vapor annealing" (SVA). ${ }^{14-}$ ${ }^{17}$ In general, interpenetrating networks with $10-15 \mathrm{~nm}$-sized domains are considered optimum for charge generation, transport and extraction, but other structural factors such as domain purity (often paired with crystallinity), ${ }^{18}$ relative molecular arrangements and orientations, ${ }^{19}$ and vertical phase-separation patterns are also known to impact $\mathrm{BHJ}$ device performance. ${ }^{20}$

Of all optimization protocols known to influence $\mathrm{BHJ}$ morphology, SVA treatments are currently being described as being especially relevant to $\mathrm{SM}$ donor-fullerene $\mathrm{BHJ}$ solar cells. ${ }^{1}$ In those instances, the active layer is exposed to an environment saturated in solvent vapors that diffuse into the organics, inducing a local and/or mesoscale reorganization of the molecular donor and acceptor counterparts. Factors that affect the effectiveness of the SVA treatment include (i) the solubility of the molecular donor and/or acceptor, (ii) the partial pressure of the selected solvent, and (iii) the time of exposure. ${ }^{14,16}$ While SVA protocols are proving useful in the optimization of the SM donor-fullerene $\mathrm{BHJ}$ morphologies and efficiencies, ${ }^{21-23}$ a better understanding of the direct correlations between solvent vapor exposure, morphological changes, crystallization, and carrier effects across the active layer (i.e. charge generation, transport, recombination and extraction) should be established in order to continue improving beyond currently reported efficiencies.

In our initial work, we described the rational design approach that led to the development of the SM donor BDTQdC (Chart 1) -a particularly efficient material system in $\mathrm{BHJ}$ solar cells with the fullerene acceptor $\mathrm{PC}_{71} \mathrm{BM}^{24}$ When subjected to SVA conditions, BDTQdC-based BHJ solar cells see their PCE increase from a 
modest $\boldsymbol{c a}$. $2.5 \%$ ("as-cast" values) to $>8 \%$, with some important apparent morphological changes. ${ }^{24}$ In the present contribution, we examine the systemic influence of the SVA protocol based on chloroform vapor employed in the optimization of $\mathrm{BHJ}$ device performance for the model system $\mathrm{BDT}[2 \mathrm{~F}] \mathrm{QdC}: \mathrm{PC}_{71} \mathrm{BM}$ and, more specifically, the impact of SVA exposure time -which is shown to mediate the selective crystallization of the SM donor $(\mathrm{BDT}[2 \mathrm{~F}] \mathrm{QdC})$ with respect to the fullerene acceptor $\left(\mathrm{PC}_{71} \mathrm{BM}\right)$, and sets the lengthscale of the phase-separated network. As the morphology evolves across the active layer, we demonstrate that SVA-mediated crystallization effects direct charge generation, recombination and extraction in the SM donor-fullerene $\mathrm{BHJ}$ solar cells, to which connects device performance figures. Our systematic examinations combine a large span of characterization techniques that provide direct insight into morphological and carrier effects, including: in-situ UV-vis absorption, high-resolution transmission electron microscopy (HR-TEM), atomic force microscopy (AFM), photoluminescence $(\mathrm{PL})$ quenching analyses, transient photocurrent (TPC) measurements, X-ray diffraction (XRD) and timedelayed collection field (TDCF).

\section{Results and Discussion Material Properties}

The solution and thin film UV-vis spectra overlaid in Figure 1a show a significant bathochromic shift on going from solution to the thin film -an observation indicating that aggregation, typically promoted by $\pi-\pi$ interactions, takes place upon solidification of the SM donor in films. ${ }^{25,26}$ The absorption of the thin films have two main spectral features, an absorption band at high energy (centered around $430 \mathrm{~nm}$ ) and a lower-energy band consisting of three apparent "peaks" (convoluted) respectively at $c a .575 \mathrm{~nm}, 630 \mathrm{~nm}$ and $688 \mathrm{~nm}$. Referring to previous work, ${ }^{5,27,28}$ we assign the $688 \mathrm{~nm}$ peak to the $A_{0-0}$ transition and the $630 \mathrm{~nm}$ peak to the $A_{0-1}$ transition. Figure $1 \mathrm{~b}$ emphasizes the temperature-dependent spectral variations observed in thin films of $\mathrm{BDT}[2 \mathrm{~F}] \mathrm{QdC}$ as the layer is subjected to a gradual elevation of temperature (up to $180^{\circ} \mathrm{C}$ ) (cf. experimental details in the SI). Looking at the intensity ratio of the 2 vibronic peaks $\mathrm{A}_{0-0}$ and $\mathrm{A}_{0-1}$ : while the film temperature increases, the ratio $\mathrm{I}_{\mathrm{A} 0-0} / \mathrm{I}_{\mathrm{A} 0-1}$ decreases, and a blue-

Chart 1. Molecular Structures of the SM Donor BDT $[2 \mathrm{~F}] \mathrm{QdC}$ and the Fullerene Acceptor $\mathrm{PC}_{71} \mathrm{BM}$.

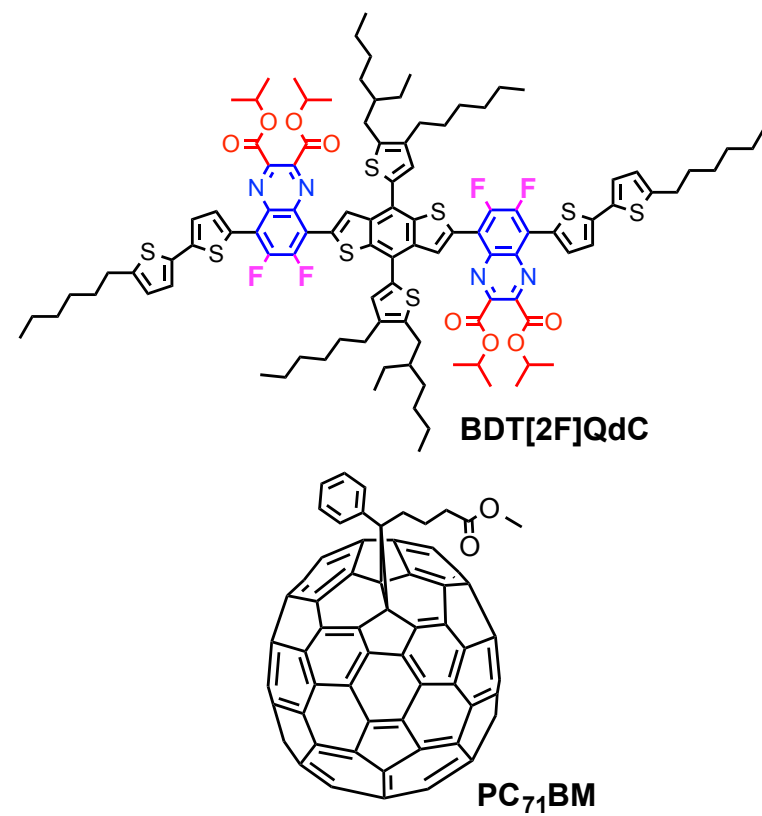

shift of the $\mathrm{A}_{0-0}$ maximum intensity is observed. The evolution of the ratio $\mathrm{I}_{\mathrm{AO}-0} / \mathrm{I}_{\mathrm{A} 0-1}$ combined with the blue-shift observed is consistent with the fact that thermal heating induces molecular disorder and weaken $\pi-\pi$ stacking interactions. ${ }^{28-30}$ Considering the remarkable variation in UV-vis absorption patterns shown in Figure $1 \mathrm{c}$ for the BDT[2F]QdC:PC ${ }_{71} \mathrm{BM}$ blend films (1:1) subjected to solvent vapors (SVA), it is evident that molecular reorganization effects are occurring across the films. Compared to the neat film, the spectrum of the BDT[2F]QdC:PC $\mathrm{PC}_{71} \mathrm{BM}$ blend in "as-cast" film shows only weak vibronic features and the $0-0$ peak around $680 \mathrm{~nm}$ is absent. In other words, the presence of $\mathrm{PC}_{71} \mathrm{BM}$ in the blend films suppresses the propensity of ordered BDT[2F]QdC aggregates to form. This behavior is consistent with prior reports depicting a reduction in overall crystallinity with the incorporation of fullerenes (commonly referred to as "vitrification" process), resulting in the formation of disordered mixed phases within the donoracceptor blends. ${ }^{31-33}$ In contrast, upon solvent exposure for 20 s, the features observed in the UV-vis spectrum of the blend film are common to those of the neat film: prominent $\mathrm{A}_{0-0}$ and $\mathrm{A}_{0-1}$ peaks. Those observations are consistent with the occurrence of pronounced morphological changes during the SVA process. In light of those analyses, we turned to a more involved study of the aggregation kinetics, performing in-situ UV-vis absorption measurements on the $\mathrm{BDT}[2 \mathrm{~F}] \mathrm{QdC}: \mathrm{PC}_{71} \mathrm{BM}$ blend films during the first 120 seconds of solvent exposure. The outcome of this study is presented in Figure 1d (full spectrum in Figure S1). In this experiment, the blend film was placed inside the Petri dish saturated in solvent vapor, and the transmission of a white light passing through the films was measured with a time resolution of two spectra per second (cf. further experimental details in the SI, section 2; for consistency, the setup used was the same as that later employed in the later device sections). Here, we note that SVA time is provided on a logarithmic scale because most morphological changes occur during the first 20 seconds. 


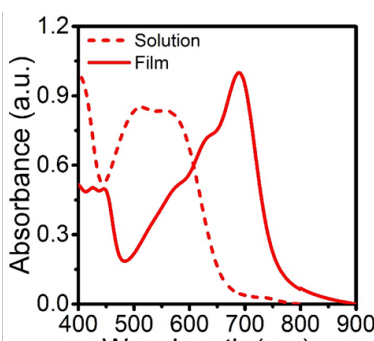

(a)
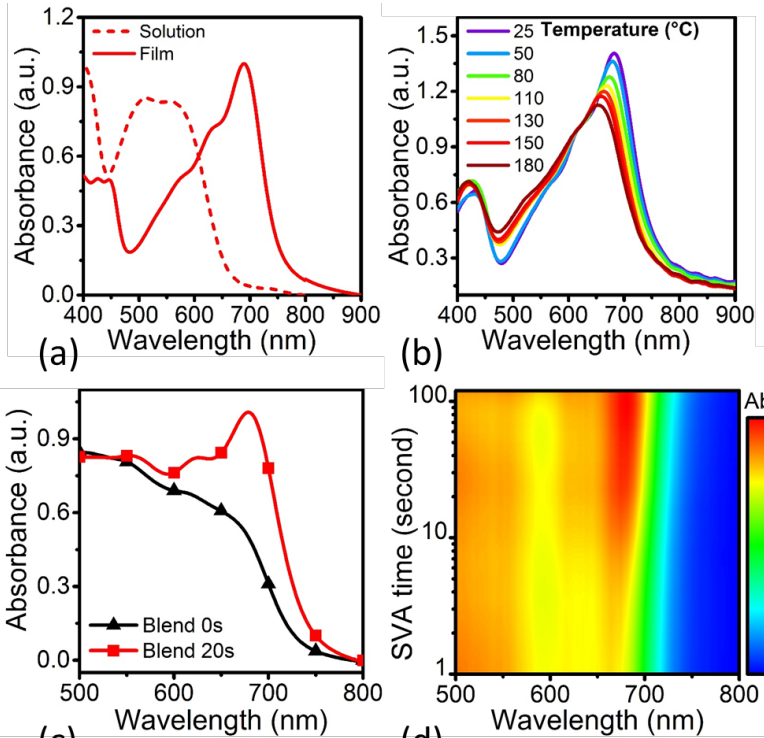

(c)

(b)

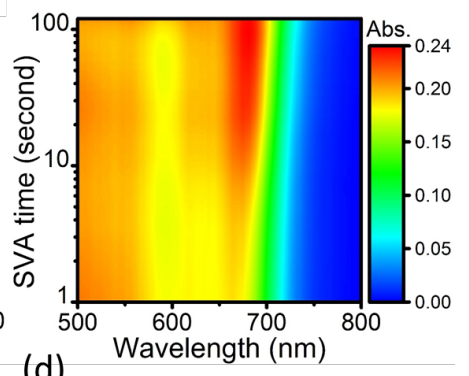

(d)

Figure 1. (a) Superimposed solution and thin-film UV-vis absorption spectra for BDT[2F]QdC (normalized). (b) Temperature-dependent thin-film UV-vis absorption for BDT[2F]QdC subjected to increasing temperatures over the range $25-180^{\circ} \mathrm{C}$. (c) UV-Vis absorption of $\mathrm{BDT}[2 \mathrm{~F}] \mathrm{QdC}: \mathrm{PC}_{71} \mathrm{BM}$ blend films (1:1), "as-cast" and after 20s chloroform (CF) vapor exposure. (d) In-situ absorbance of $\mathrm{BDT}[2 \mathrm{~F}] \mathrm{QdC}: \mathrm{PC}_{{ }_{71} \mathrm{BM}}$ blend films (1:1) vs. solvent vapor annealing (SVA) exposure time.

Within the first 20s, the 0-0 peak emerges, and its maximum peak intensity red-shifts to some extent upon solvent exposure. Since the intensity of the 0-1 peak increases only slightly during this time, the overall ratio $\mathrm{I}_{\mathrm{AO}-\mathrm{O}} / \mathrm{I}_{\mathrm{A} 0-1}$ increases substantially, indicating that structural order is developing in the film, along with $\pi-\pi$ aggregation. From 20 to 120 seconds, the UV-vis spectrum of the $\mathrm{BDT}[2 \mathrm{~F}] \mathrm{QdC}: \mathrm{PC}_{71} \mathrm{BM}$ blend film shows only minor changes over time, suggesting that any further crystallization may be occurring from the merging of existing crystallites.

\section{Device Examinations}

Solution-processed thin film BHJ solar cells were fabricated using the conventional device architecture ITO/PEDOT:PSS/ $\mathrm{BDT}[2 \mathrm{~F}] \mathrm{QdC}: \mathrm{PC}_{71} \mathrm{BM} / \mathrm{Ca} / \mathrm{Al}$ (device area: $0.1 \mathrm{~cm}^{2}$ ), and were tested under simulated AM1.5G illumination $\left(100 \mathrm{~mW} / \mathrm{cm}^{2}\right)$; the detailed experimental protocol is provided in the SI (see section 1). Table 1 summarizes the figures of merit of the $\mathrm{BHJ}$ devices made from $\mathrm{BDT}[2 \mathrm{~F}] \mathrm{QdC}$ and $\mathrm{PC}_{71} \mathrm{BM}$, where the active layers are treated with various SVA times (up to 120 seconds); the $J-V$ curves of the corresponding devices are shown in Figure 2a. As shown in Table 1, the reference "as-cast" device shows an open-circuit voltage $\left(V_{\text {oc }}\right)$ of $0.96 \mathrm{~V}$ and only modest short-circuit current $(J s c)$ and fill-factor $(F F)$ values of $6.9 \mathrm{~mA} / \mathrm{cm}^{2}$ and $33 \%$, respectively. Within the first 20s of SVA treatment, both the $\boldsymbol{J}_{\mathrm{sc}}$ and $\boldsymbol{F} \boldsymbol{F}$ increase significantly, reaching $11.4 \mathrm{~mA} / \mathrm{cm}^{2}$ and $66 \%$, respectively. The power conversion efficiency (PCE) upon optimal solvent annealing time

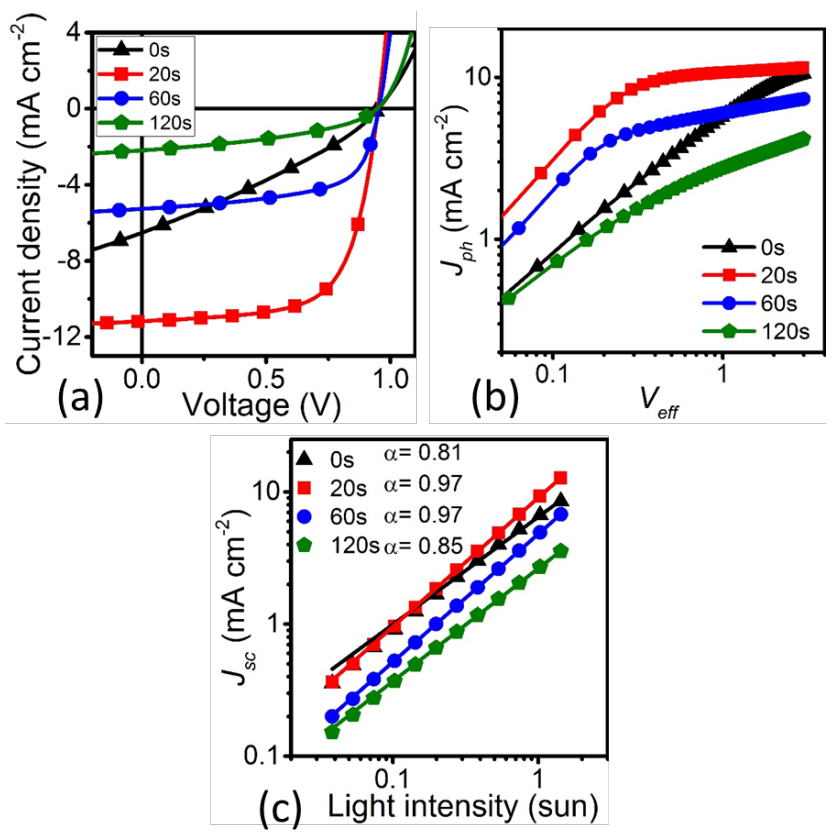

Figure 2. (a) Characteristic $J-V$ curves of $B H J$ solar cells made from $\mathrm{BDT}[2 \mathrm{~F}] \mathrm{QdC}: \mathrm{PC}_{71} \mathrm{BM}$ active layers subjected to various SVA times. (b) Photocurrent $\left(J_{p h}\right)$ vs. effective applied voltage $V_{\text {eff }}=\left(V_{0}-V\right)$. (c) $J_{S C}$ vs. light intensity of $\mathrm{BDT}[2 \mathrm{~F}] \mathrm{QdC}: \mathrm{PC}_{71} \mathrm{BM}$. The solid lines correspond to fits to the data according to $J_{S C} \propto I^{\alpha}$. Analyses performed via a white LED with a maximum irradiance of $200 \mathrm{~mW} / \mathrm{cm}^{2}$. In accounting for spectral mismatch, $70 \%$ of the maximum irradiance of the white-light LED was used to reproduce the $J_{s c}$ values achieved under standard AM1.5G solar illumination $\left(100 \mathrm{~mW} / \mathrm{cm}^{2}\right)$.

Table 1. Summary of PV device performance parameters for $\mathrm{BDT}[2 \mathrm{~F}] \mathrm{QdC}: \mathrm{PC}_{71} \mathrm{BM}$ active layers subjected to various solvent vapor annealing (SVA) times.

\begin{tabular}{cccccc}
\hline SVA time & $\boldsymbol{V}_{\text {OC }}$ & Jsc & FF & $\begin{array}{c}\text { Avg. } \\
\text { PCE }^{a}\end{array}$ & Max. PCE \\
{$[\mathbf{s}]$} & {$[\mathbf{V}]$} & {$\left[\mathbf{m A} / \mathbf{c m}^{2}\right]$} & {$[\%]$} & {$[\%]$} & {$[\%]$} \\
\hline 0 & 0.96 & 6.9 & 33 & 2.2 & 2.3 \\
5 & 0.95 & 9.5 & 38 & 3.4 & 3.7 \\
20 & 0.94 & 11.4 & 66 & 7.1 & 7.2 \\
60 & 0.94 & 5.5 & 58 & 3.1 & 3.2 \\
120 & 0.94 & 2.1 & 36 & 0.7 & 0.9 \\
\hline
\end{tabular}

${ }^{a}$ Additional device statistics are provided in Table S1 in the Supporting Information.

peaks to 7.1\% (Avg.; 7.3\% Max.). Upon longer solvent exposure times, the $J_{s c}$ decreases and drops to $5.5 \mathrm{~mA} / \mathrm{cm}^{2}$ after $60 \mathrm{~s}$, and down to $1.8 \mathrm{~mA} / \mathrm{cm}^{2}$ after 120 s. The $F F$ also decreases from $66 \%$ for "optimized" devices to $36 \%$ after 120 s. It is worth noting that the $\boldsymbol{F F}$ remains as high as $58 \%$ after 60 s. After 120 s of SVA treatment, the PCE drops to $0.7 \%$, and longer solvent annealing times result in partial active layer deterioration, leaving partially uncovered areas across the substrate. Clearly, the SVA treatment plays a determining role, setting the conditions for high $\boldsymbol{J}_{s c}$ and $F \boldsymbol{F}$ values, but can also be detrimental to device operation. Assuming that the SVA exposure times directly impacts active layer morphology, an 
indirect examination of the internal electric field across the $\mathrm{BHJ}$ solar cells should reveal significant variations in active layers exposed to various SVA times.

To understand the role of the internal electric field on the device current we analysed the photocurrent as a function of the effective voltage $;^{34,35}$ data presented in Figure $2 \mathrm{~b}$. The photocurrent $J_{p h}$ is given by the current measured under light minus the current measured in the dark. The effective applied voltage $V_{\text {eff }}$ is defined as the voltage $V_{0}$ for $J_{p h}=0$ minus the applied voltage $\boldsymbol{V}$. For BHJ devices subjected to $20 \mathrm{~s}$ and $60 \mathrm{~s}$ SVA, $J_{p h}$ increases rapidly at low voltages and saturates at an effective voltage of $c a .0 .3 \mathrm{~V}$. The early onset of saturation of the photocurrent observed in those devices indicates that the internal electric field plays a minor role during charge extraction, and that charges are efficiently extracted at the electrodes. The distinct values of saturated current reflect the difference in $J_{s c}$ between the two devices (see Table 1). In contrast, "as-cast" devices do not show this saturation regime as the photocurrent keeps increasing with applied voltage. Interestingly, at significantly higher effective voltage $(>3 \mathrm{~V})$, the "as-cast" device and the optimized devices have similar photocurrents, indicating that the modest $J_{s c}$ measured for the as-cast device does not originate from charge generation issues, but rather from a poor extraction of carrier at the electrode interfaces. If not assisted by a strong electric field, the charges recombine before reaching the electrodes. The photocurrent measured for the BHJ devices subjected to $120 \mathrm{~s}$ SVA also show a continuous dependence on voltage, with no visible saturation regime. In this instance, the photocurrent remains low at high voltages (3-4 $\left.\mathrm{mA} \mathrm{cm}^{-2}\right)$, even though the electric field increases charge collection to some extent.

In order to quantify the extent of bimolecular recombination occurring in active layers subjected to varying SVA times, we tested the BHJ solar cells under various light illumination intensities. It has been shown that the extent of bimolecular recombination can be estimated looking at the dependency of different illumination conditions on the device $J_{s c .}{ }^{36}$ The dependence of the $J_{s c}$ is described by $J_{s c} \propto I^{\alpha}$. Here, a value of $\alpha$ close to unity indicates a regime where bimolecular recombination losses can be considered negligible, whereas smaller $\alpha$ values indicate a competition between recombination and carrier extraction. ${ }^{37}$ Figure $2 \mathrm{c}$ shows the $J_{s c}$ versus light intensity data for various SVA times, (all $J-V$ curves are provided in Figure S2). For the as-cast device (no SVA treatment) the fit yields $\alpha=0.81$, indicating substantial bimolecular recombination losses. After SVA exposure for 20s and 60s, $\alpha=0.97$, suggesting that bimolecular recombination gets significantly reduced. Longer SVA treatments of 120 s result in $\alpha=0.85$, indicating that bimolecular recombination significantly limits device efficiency for longer exposure times as well.

In several prior studies, ${ }^{14,16,23}$ SVA treatments have been described as inducing crystallization and changing the morphology of the $\mathrm{BHJ}$ blend films. Thus, one may assume that crystallization effects occurring in $\mathrm{BDT}[2 \mathrm{~F}] \mathrm{QdC}: \mathrm{PC}_{{ }_{71}} \mathrm{BM}$ active layers subjected to SVA may also be at the origin of significant macroscopic changes in thin-film morphology. Here, active layer morphologies were analyzed via bright-field transmission electron microscopy (TEM) and atomic force microscopy (AFM); results shown in Figure 3. Figure 3 shows the blend morphologies obtained upon exposure to SVA treatments of varying duration. Donor- and acceptor-rich domains (bright and darker regions, respectively) appear rather intermixed in the as-cast films (Figure 3a), and domain sizes can be inferred as $<10 \mathrm{~nm}$. During solvent exposure, the $\mathrm{BHJ}$ reorganizes and the size of the domains increases. After 20s, time after which the "optimized" thin-film condition is obtained, the size of the do- 
mains increases to $15-20 \mathrm{~nm}$-a favorable domain size range allowing excitons to reach $\mathrm{D} / \mathrm{A}$ interfaces, and favoring carrier generation and percolation required for efficient transport and extraction. Upon further solvent exposure, the donor-rich domains form needle-like morphologies across the active layer. By direct imaging, the widths of these apparent crystalline features can be inferred as ca. 35,95 and $600 \mathrm{~nm}$ (avg. widths) for SVA-treated films exposed for 30,60 and 120s respectively. Large domains (>30nm) are not desirable from a charge generation standpoint, as excitons tend to decay before reaching the donor-acceptor interface. After 120 s of solvent exposure, the SM donor crystallites are overly grown (as large as film thickness) and the active layers no longer form $\mathrm{BHJ}$ networks. These severe aggregation patterns obtained upon prolonged solvent exposure have commonly been held responsible for the loss of photocurrent. ${ }^{21}$ AFM images and root mean square (RMS) roughness estimates are provided in Figure S3. As-cast BHJ thin films are smooth RMS estimates of ca. $0.5 \mathrm{~nm}$. RMS values then gradually increase for active layers exposed to longer SVA times. For films said "optimized", RMS values remain low at ca. 1.3 $\mathrm{nm}$. RMS estimates then rise quickly to values as high as 19.8 and $45.6 \mathrm{~nm}$ for SVA times of 60 and 120s, respectively. Those increases in RMS roughness are consistent with the TEM data indicative of a substantial growth of the SM donor-rich domains.

To examine the effect of domain size on charge generation and device photocurrent, we analyzed the propensity of excitons to reach the donor-acceptor interfaces via photoluminescence quenching (PLQ) measurements. Figure $3 \mathrm{f}$ shows the PLQ data for films exposed to various SVA times (raw data provided in Figure S4). The high PLQvalue of 93\% measured for the as-cast BHJ thin film is consistent with the short domain lengthscale observed for the same films via high-resolution TEM (Figure 3), and indicates that excitons are effectively reaching the interface. With longer SVA times, the SM donor-rich phases grow and the PLQ values drop to some extent. It is worth noting that "optimized" films undergo a slight reduction in PLQ value of 5-10\% compared to as-cast films, but here we note that the corresponding devices still show increased current density, suggesting that the somewhat increased exciton quenching process does not significantly affect charge generation at this stage. However, longer solvent exposure times lead to significantly larger domain sizes, which in turn substantially decrease the PLQvalues to 58 and $53 \%$ for 60 and 120s SVA times, respectively. These low PLQ values are consistent with pronounced exciton decay reflected in reduced charge generation (in agreement with the device current densities of $<3 \mathrm{~mA} / \mathrm{cm}^{2}$ ). Correlated with the TEM analyses (Figure 3), the PLQ data is also pointing to the high purity of the SM donor crystallites forming on longer SVA times (needle-like domains).

Earlier grazing incidence wide-angle X-ray scattering (GIWAXS) experiments showed that the SM donor BDT[2F]QdC tends to crystallize in neat films, adopting edge-on orientations with respect to the substrate with intermolecular $\pi-\pi$ spacings of ca. $3.68 \AA .{ }^{24}$ However, in blends with $\mathrm{PC}_{71} \mathrm{BM}$, the $\mathrm{SM}$ donor does not maintain the same degree of structural order and crystallites with mixed orientations prevail. ${ }^{24}$ Solvent exposure induces more crystallinity, as reflected by the higher-order lamellar reflections and more pronounced $\pi-\pi$ stacking arcs visible in the GIWAXS spectra. In Figure 3g,h, we use specular X-ray diffraction to provide further insights into the aggregation pattern of the SM donor in both neat and blend films with $\mathrm{PC}_{71} \mathrm{BM}$ subjected to various SVA times. In the specular reflection data, out-of-plane diffraction peaks are observed where the (100), (200) and (300) reflections are resolved. The specular data is also consistent with the fact that $\mathrm{BDT}[2 \mathrm{~F}] \mathrm{QdC}$ tends to adopt an edge-on configuration, and from the data a lamellar spacing of $1.70 \mathrm{~nm}$ can be inferred. Figure $3 \mathrm{~h}$ shows that the high crystallinity of the neat films is not maintained as $\mathrm{BDT}[2 \mathrm{~F}] \mathrm{QdC}$ is mixed with $\mathrm{PC}_{71} \mathrm{BM}$ in as-cast active layers, with only a relatively broad first-order reflection showing on the $\mathrm{X}$ ray pattern, while the corresponding diffraction peak intensity is greatly reduced ("vitrification" process). This observation is consistent with the features described earlier from the absorption spectra of the BDT[2F] QdC:PC ${ }_{71} \mathrm{BM}$ blend films (Fig. 1c). Upon solvent annealing of the active layer, the intensity of the first-order lamellar peak increases and its intensity rises, indicating the coherence length of the SM donor gradually increases during the first 40s. After 60s SVA time, the XRD patterns become comparable to that of the neat SM donor with high-intensity higher-order reflections indicative of the significantly higher structural organization achieved in the $\mathrm{BHJ}$ blend films.

Important morphological differences between films often correlate with distinct charge transport patterns and, in SM-based $\mathrm{BHJ}$ solar cells with fullerene acceptors, the occurrence of crystalline aggregates -in which carrier transport proceeds by intermolecular charge transfer events- only exacerbates the morphological dependence. The carrier mobilities of the various active layers subjected to distinct SVA treatment durations were determined from the MIS-CELIV method; ${ }^{38}$ results reported in Figure 4 (see experimental details in the SI, section 7). As shown in Figure 4, the hole and electron mobilities of $\boldsymbol{a s}$-cast devices

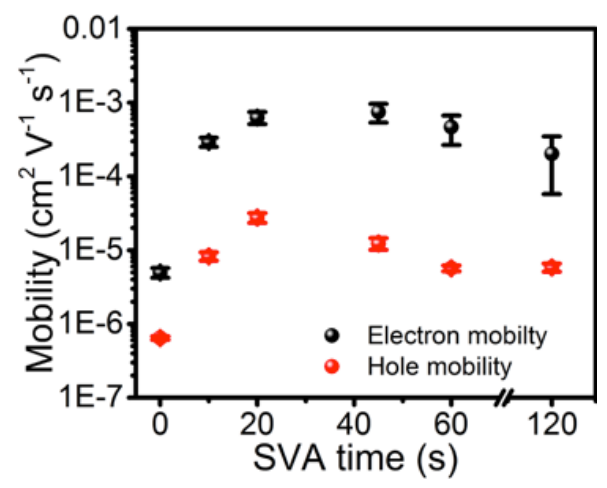

Figure 4. Hole- and electron-only MIS-CELIV mobility of the active layers exposed to various SVA times. The error bars represent the standard deviations (estimated from at least 3 devices). MIS-CELIV dark-current transients are provided in Figure S5 (electrons) and Figure S6 (holes).

are rather modest: $6.5 \times 10^{-7}$ and $5.0 \times 10^{-6} \mathrm{~cm}^{2} \mathrm{~V}^{-1} \mathrm{~s}^{-1}$, respectively. However, those figures increase rapidly within the first 20s of SVA treatment. Beyond 20s, the hole and electron mobilities reach $2.8 \times$ $10^{-5} \mathrm{~cm}^{2} \mathrm{~V}^{-1} \mathrm{~s}^{-1}$ and $6.3 \times 10^{-4} \mathrm{~cm}^{2} \mathrm{~V}^{-1} \mathrm{~s}^{-1}$, respectively -representing significant increases of $c a$. $\mathrm{X} 40$ and $\mathrm{x} 140$ (respectively) compared to the as-cast films. The slight mobility reductions estimated for active layers subjected to longer SVA exposure times are likely the result of the less continuous $\mathrm{BHJ}$ networks as pronounced crystalline aggregation takes place in the films. Low and/or unbalanced 
mobilities correlated to morphological factors can induce spacecharge effects that impact $\mathrm{BHJ}$ solar cell performance. ${ }^{21,39}$

To examine the role of morphology-related limiting factors on space-charge effects, we turned to transient photocurrent (TPC) measurements. In recent work, TPC measurements have been used to investigate space-charge effects, carrier trapping and de-trapping in polymer-fullerene, ${ }^{40,41}$ "all-polymer" ${ }^{42}$ and hybrid ${ }^{43} \mathrm{BHJ}$ solar cells. During the measurement, devices held in the dark are subjected to a long light pulse $(200 \mu \mathrm{s})$ allowing the short-circuit current to reach its steady-state value, and various light intensities are scanned (the LED intensity corresponding to "one sun equivalent" produces a device current equivalent to the one obtained upon illumination via solar simulator). In general, the rise/fall behavior of the photocurrent is described with respect to two components, a fast initial rise/fall component followed by a second component of slower dynamics. Figures 5a-d (normalized at $190 \mu \mathrm{s})$ and Figure S7 (raw data) show the transient short-circuit current for the devices subjected to the various, aforementioned SVA times. In the ascast active layers, the rise time (defined as the time taken to go from 10 to $90 \%$ current intensity) drops from 39 to $1.2 \mu$ s when the light intensity increases from 0.14 to 1.43 sun. With increasing light intensity, the fast rise of the current evolves into a transient peak before leveling off to the steady state $J$ sc. In parallel, the slow component gets suppressed as light intensity increases -an observation that can reflect the filling of trap states at higher intensities ${ }^{44}$ and/or charge density-dependent carrier mobility. ${ }^{45}$ The transient photocurrent peak observed at higher light intensities can be attributed to space-charge effects, whereby an accumulation of one carrier type occurs under the effect of mobility ratio imbalance after a few $\mu$ s, thus reducing charge generation and increasing recombination..$^{46}$ In contrast, active layers exposed either to SVA treatments for 20s and 60 s exhibit fast rise/fall times almost independent of light intensity, indicative of a favorable device behavior for which modest mobilities, carrier traps and space-charge effects are not significant limiting factors. For 120s SVA treatments, the TPC data shows a prominent transient peak within the first $50 \mu$ s; broad and weak at low intensities, the photocurrent peak sharpens and becomes more pronounced with increasing light intensity (i.e. as the number of carriers increases across the device). Vertical phase segregation and carrier-blocking layers forming at interfaces can induce this behavior. $^{47}$ Here, we think that trapped electron populations in the $\mathrm{PC}_{71} \mathrm{BM}$-rich domains (higher mobility phase) steadily build up, inducing trap-mediated space-charge characteristics that promotes recombination and suppresses charge generation.

The origin of the space-charge effects and the occurrence of charge build-ups can be confirmed by examining the voltage dependence of the transient current. By applying a voltage bias, the internal field in the device varies; if the internal field increases, the drift of the charges is promoted (reverse bias) and if the internal field gets reduced, the open-circuit condition is approached and carrier recombination increases (forward bias). Figure 5e-h shows the corresponding data for 1 sun equivalent (normalized in Figure S8); the applied bias being scanned from $-0.75 \mathrm{~V}$ to $+0.75 \mathrm{~V}$. As illustrated in Figure 5, the as-cast device sees its current vary greatly (from $2 \mathrm{~mA}$ to $10 \mathrm{~mA}$ ) upon scanning of the applied bias observation consistent with the low FF of the device indicative of a notable recombination regime. Given the low carrier mobilities estimated earlier for the as-cast device, a large reverse bias effectively promotes the drift of the charges and favors hole collection (suppressing the space-charge effects seen at short-circuit). In contrast, near the open-circuit voltage, the internal field is low and carrier collection becomes less effective. As holes accumulate (spacecharge), the transient peak becomes more pronounced and the collection current is suppressed. On the other hand, devices subjected to 40s and 60s SVA treatments show minimal-to-no dependence on the applied voltage. For those devices, the shape of the transient current is practically independent of the applied voltage (also see Fig. S8 b-c). Importantly, Figure 5h shows that active layers exposed to longer SVA times (here, 120s) suffer from a dependence on the applied bias that resembles that of as-cast devices. Those results can be correlated to those discussed earlier from Figure 3, showing the occurrence of overgrown crystallites with domain sizes far greater than 

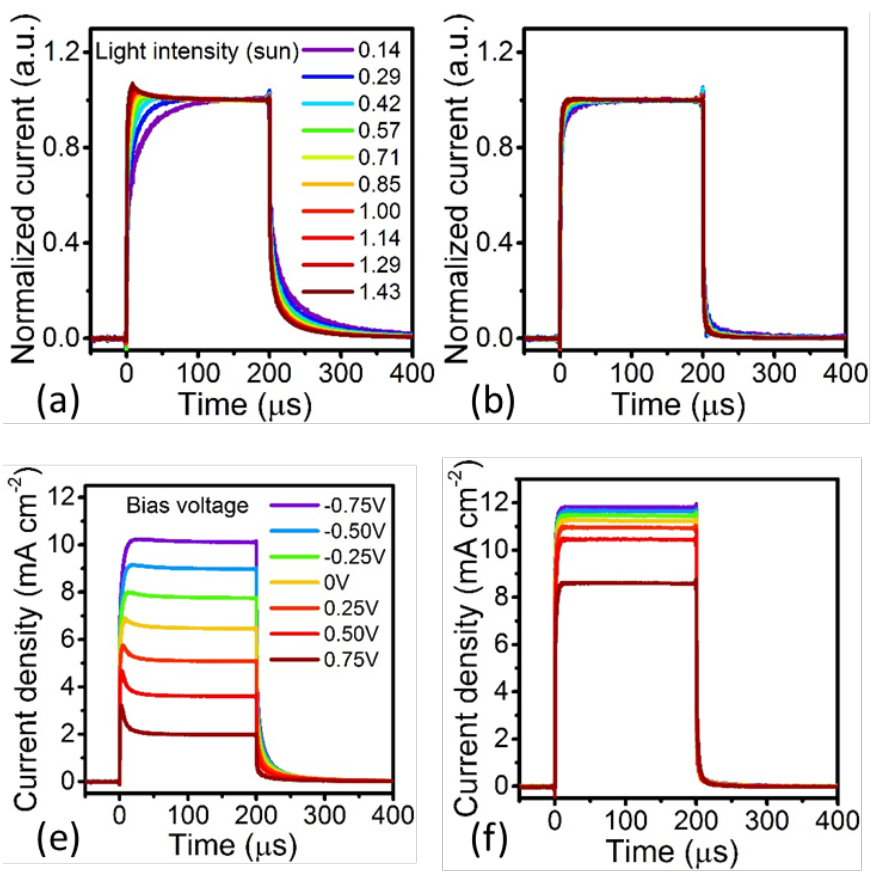
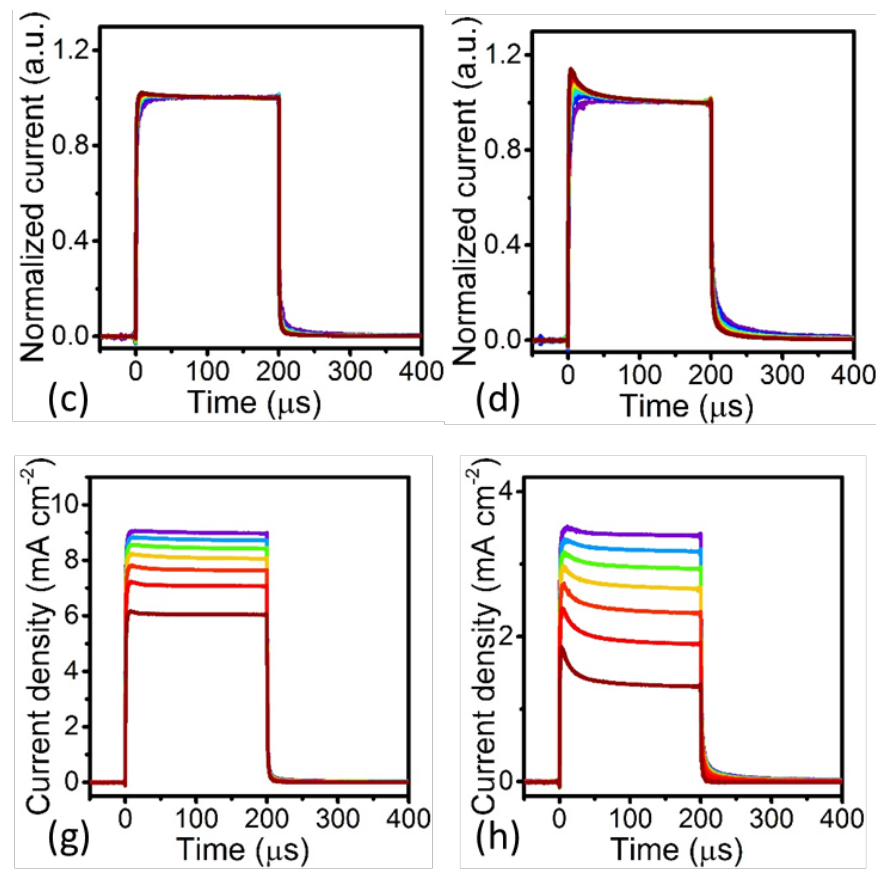

Figure 5. Transient $J_{S C}$ (normalized) in response to a $200 \mu$ s white light (LED) pulse for various light intensities (given in sun equivalent) and for BHJ active layers subjected to varying SVA exposure times: (a) "as-cast" (0s), (b) 20s, (c) 60s, and (d) 120 s. Transient current in response to the 200 $\mu$ s white light (LED) pulse at 1 sun equivalent, for various voltage biases applied and for BHJ active layers subjected to varying SVA exposure times: (e) "as-cast" (0s), (f) 20s, (g) 60s, and (h) 120s.

the diffusion length of excitons. In this case (120s SVA time), the phase-separation lengthscale is such that trapped carriers cannot be efficiently extracted even under large reverse voltages. Those accumulate in the active layer, changing the local electric field, increase recombination effects and suppressing charge generation.

To examine the impact of the electric field on geminate recombination and charge generation, we turned to time delayed collection field (TDCF) experiments. In prior studies using TDCF experiments to probe the influence of the electric field on charge generation: Knipert et al. described a charge generation process independent of the electric field in "as-cast" and optimized P3HT:PC ${ }_{71} \mathrm{BM}$ blend films; ${ }^{48,}{ }^{49}$ considering various polymer:PC ${ }_{71} \mathrm{BM}$ systems, Albrecht et al. emphasized an apparent interdependence between chemical structure, optimization protocol and dependence of charge generation on the electric field; $;{ }^{50,51}$ Foertig et al. correlated the use of the processing additive DIO, morphological changes in the blend films, and an apparent suppression of the dependence of charge generation on the electric field; ${ }^{52}$ and Proctor et al. related the use of DIO to the reduction of geminate recombination in a SM-based blend sytem..$^{53}$ In TDCF analyses, the devices are subjected to a pre-bias $\left(\mathrm{V}_{\mathrm{pre}}\right)$ and illuminated with a short laser pulse. After a certain delay time ( $\left.t_{d}\right)$, a large reverse bias is applied to the solar cell to collect all the remaining carriers. The transient current is integrated to determine the total of collected charges $\left(Q_{\text {tot }}\right)$. The fluence of the laser pulse is low enough that non-geminate recombination remains negligible while geminate recombination represents the main loss channel. Measuring $Q_{\text {tot }}$ as a function of the pre-bias can be seen as a direct measurement of the voltage dependence of geminate recombination. First, the pulse fluence was varied from 0.02 to $1.5 \mu \mathrm{J} . \mathrm{cm}^{-2}$ in order to determine the regime where the relation between light intensity and the col- lected charges is linear (Figure S9). The excitation wavelength was chosen to be $532 \mathrm{~nm}$, noting that at this wavelength the absorption of the blend does not change during the SVA treatment. The measurements were performed with a delay time of $10 \mathrm{~ns}$ and a $\mathrm{V}_{\text {coll }}$ of $4 \mathrm{~V}$; results shown in Figure 6. Interestingly, for the non-optimized as-cast device, $\mathrm{Q}_{\text {tot }}$ is independent of $\mathrm{V}_{\mathrm{pre}}$. The charge generation is independent of the pre-bias voltage. Devices for which the active layer was subjected to 20s SVA exposure time show a weak dependence of the charge generation on the pre-bias $V_{\text {pre. }}$ Geminate losses at open-circuit condition are $8 \%$ higher than the losses incurred at $-2.5 \mathrm{~V}$. For active layers exposed to 60s SVA times, the dependence is even stronger with geminate losses $18 \%$ higher at open-circuit condition compared to the loss incurred at $-2.5 \mathrm{~V}$. Here, it is worth noting that these results differ from those obtained earlier by Proctor et al. who found that geminate recombination in as-cast devices can be strongly voltage-dependent, whereas "optimized" devices cast using DIO as the processing additive did not show the same dependence. ${ }^{53}$ In their study, the reduced field dependence was correlated to the highly ordered crystalline domains obtained in active layers cast with DIO. Since the solvent vapor annealing process also increases domain sizes while promoting phase crystallinity, a similar trend could have been expected. In light of those differing outcomes, we conclude that the relative crystallinity of the domains alone cannot explain the voltagedependence of charge generation. Other factors difficult to probe and beyond the scope of this study could have a contribution to the voltage-dependence: (i) molecular orientation at the donoracceptor interface, (ii) the concomitance of ordered and disordered domains, and (iii) the presence of the so-called "mixed" phase. In $\mathrm{BDT}[2 \mathrm{~F}] \mathrm{QdC}: \mathrm{PC}_{71} \mathrm{BM}$ active layers, the presence/absence of mixed phase may be at the origin of the behavior observed. Based 
on direct TEM imaging and considering the crystallization process of $\mathrm{BDT}[2 \mathrm{~F}] \mathrm{QdC}$ mediated by SVA treatment, the interface between the donor
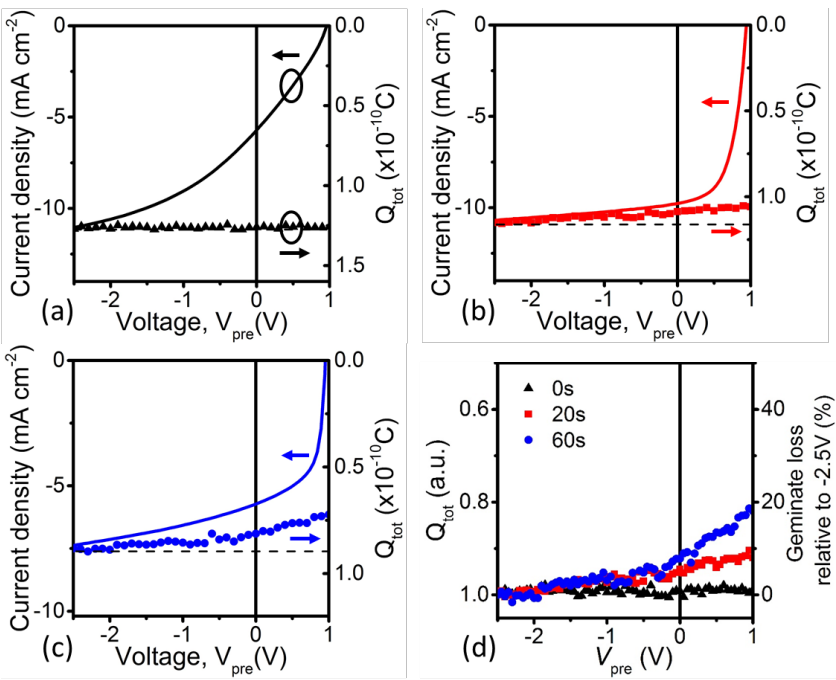

Figure 6. $J-V$ curves (left axis) and voltage dependence of the total charge extracted as probed by TDCF experiment (right axis) for BHJ active layers subjected to varying SVA exposure times: (a) "as-cast" (0s), (b) 20s, (c) 60s. (d) Total extracted charge data normalized to $\mathrm{Q}_{\text {tot }}$ at $-2.5 \mathrm{~V}$. The right axis provides the percentage of photogenerated carriers lost due to geminate recombination relative to the loss incurred at $-2.5 \mathrm{~V}$.

and the acceptor domains is excepted to be sharp and abrupt -a situation likely to result in the reduction of mixed-phase at those interfaces. The mixed phase is known to be beneficial to charge separation and to suppress carrier recombination in $\mathrm{BHJ}$ solar cells. ${ }^{19,54,55}$

\section{Additional Remarks}

As-cast active layers show a high degree of intermixed donorand acceptor-rich domains -a situation that can lessen the level of interconnection within donor and acceptor phases through molecular intercalation (e.g. fullerenes isolating SM donors and disrupting their packing/aggregation propensity) and, in turn, disfavor efficient percolation pathways for charge transport. As the UV-Vis data (Fig. 1) lacks the footprint of structural order through aggregation, the XRD pattern of the as-cast blend (Fig. 3h) indicates that the structural order present in the neat films of SM donor is not preserved in the blend film. In contrast with polymer-fullerene $\mathrm{BHJ}$ solar cells, where charge transport occurs mainly through $\pi$ conjugated main-chain conduction pathways (including tie-chains interconnecting distant domains), carrier transport in SM donorfullerene systems is dominated by intermolecular charge transfer processes and relies on molecular interconnects. With the low degree of structural order seen in as-cast devices, the carriers mobilities are at their minimum, $\sim 10^{-7}$ and $\sim 10^{-6} \mathrm{~cm}^{2} \mathrm{~V}^{-1} \mathrm{~s}^{-1}$ for holes and electrons, respectively. In the meantime, the small domain sizes $(<10 \mathrm{~nm})$ provide a high density of D/A interfaces, favoring charge separation processes across the active layer. In those devices, charge generation is efficient and shows no sign of voltagedependence. Nevertheless, those same devices suffer from low charge collection yields. Given the limited mobilities and the non- ideal bulk morphology lacking percolation pathways, free carriers are not efficiently reaching the electrode interfaces, and tend to recombine non-geminately instead. The modest and unbalanced mobilities in as-cast devices cause an accumulation of holes (space charge effect) across the active layer that further promotes recombination and suppresses charge generation. In contrast, in SVAtreated active layers, solvent exposure induces the crystallization of the SM donor, and both domain size and crystallinity increases, impacting device performance. Upon 20s of SVA exposure, the morphology transitions from overly mixed to a favorable cocontinuous network with domains sizes of $15-20 \mathrm{~nm}$ that are adequate for charge generation and charge collection processes. As packing and structural order increase, carrier mobilities improve, promoting charge transport and charge collection. Bimolecular recombination is greatly reduced in parallel. This condition yield the best device efficiency figures, comprising the highest $J_{s c}$ and FF values achievable with $\mathrm{BDT}[2 \mathrm{~F}] \mathrm{QdC}: \mathrm{PC}_{71} \mathrm{BM}$ active layers.

On the other hand, exposing the active layer to the solvent for more extended periods of time results in a deterioration of device performance. In those instances, the SM donor forms larger-sized crystals of high purity (needle-like structures) and the morphology evolves towards domain sizes larger than the exciton diffusion length, affecting charge generation. A significant extent of excitons decay before they can reach a D/A interface and the $J_{s c}$ plummets. Thus, in 60s-SVA treated devices, while the high FF, high mobilities, fast transient behavior and low bimolecular recombination characteristics can be maintained, higher yields of exciton quenching (PLQ data, Fig. 3f) result in an overall drop in efficiency. Beyond 120s-SVA times, new morphological effects take place and the interpenetrating network model is no longer valid. SM donor domains are now sufficiently extended that their lengthscale matches the thickness of the active layer, hindering electron collection at the cathode (amongst other issues). In turn, $\mathrm{PC}_{71} \mathrm{BM}$ can be expected to segregate in the vertical direction across the $\mathrm{BHJ}$ and/or accumulate at the wrong interface (i.e. anode). Poor charge generation and extraction are at the source of the more modest efficiencies estimated for those devices, which show lower photocurrents and the characteristics of charge-trapping effects.

\section{Conclusions}

In summary, our examinations of the time-dependence of solvent vapor annealing (SVA) on SM donor-fullerene BHJ solar cell efficiency via a combination of direct morphology imaging and spectroscopy techniques establish a fundamental understanding of this important device optimization protocol. As SVA treatments induce a reorganization of the $\mathrm{BHJ}$ morphology from a highly intermixed blend with limited structural order to a structurally ordered, co-continuous network, with crystalline domains sizes of 15$20 \mathrm{~nm}$ (20s-SVA condition), charge generation and collection patterns dramatically improve. However, our analyses indicate that extended SVA times $(>60 \mathrm{~s})$ can promote excess crystallization and undermine device performance, as significant impediments arise from larger domain sizes: specifically, increased exciton quenching and detrimental extent of phase segregation between SM donor and fullerene acceptor. Since SVA treatments tend to reduce the density of mixed phase across the active layer, our findings show that it is critically important to control SVA exposure times. Over- 
all, SVA protocols represent a particularly useful tool in the optimization of SM donor-fullerene BHJ solar cells, but our study also shows that they are an efficient method to examine morphologycharge dynamics relationships in BHJ solar cells in general independently of intrinsic molecular characteristics when comparing two or more molecules.

\section{ASSOCIATED CONTENT}

\section{Supporting Information}

Experimental methods, characterization, and additional figures and tables. This material is available free of charge via the Internet at http://pubs.acs.org.

\section{AUTHOR INFORMATION}

\section{Corresponding Author}

${ }^{*}$ E-mail: pierre.beaujuge@kaust.edu.sa

\section{Notes}

The authors declare no competing financial interest.

\section{ACKNOWLEDGMENT}

This publication is based upon work supported by the King Abdullah University of Science and Technology (KAUST) Office of Sponsored Research (OSR) under Award No. CRG_R2_13_BEAU_KAUST_1. The authors acknowledge concurrent support under Baseline Research Funding from KAUST.

\section{REFERENCES}

(1) Collins, S. D.; Ran, N. A.; Heiber, M. C.; Nguyen, T.-Q., Small is Powerful: Recent Progress in Solution-Processed Small Molecule Solar Cells. Adv. Energy Mater. 2017, 7, 1602242.

(2) Cai, Y.; Huo, L.; Sun, Y., Recent Advances in Wide-Bandgap Photovoltaic Polymers. Adv. Mater. 2017, 29, 1605437.

(3) Dou, L.; Liu, Y.; Hong, Z.; Li, G.; Yang, Y., Low-Bandgap Near-IR Conjugated Polymers/Molecules for Organic Electronics. Chem. Rev. 2015, 115, 12633-12665.

(4) Roncali, J.; Leriche, P.; Blanchard, P., Molecular Materials for Organic Photovoltaics: Small is Beautiful. Adv. Mater. 2014, 26, 38213838.

(5) Wan, J.; Xu, X.; Zhang, G.; Li, Y.; Feng, K.; Peng, Q., Highly efficient halogen-free solvent processed small-molecule organic solar cells enabled by material design and device engineering. Energy Environ. Sci. 2017, 10, 1739-1745.

(6) Qiu, B.; Xue, L.; Yang, Y.; Bin, H.; Zhang, Y.; Zhang, C.; Xiao, M.; Park, K.; Morrison, W.; Zhang, Z.-G.; Li, Y., All-Small-Molecule Nonfullerene Organic Solar Cells with High Fill Factor and High Efficiency over 10\%. Chem. Mater. 2017.

(7) Lin, Y.; Wang, J.; Li, T.; Wu, Y.; Wang, C.; Han, L.; Yao, Y.; Ma, W.; Zhan, X., Efficient fullerene-free organic solar cells based on fusedring oligomer molecules. J. Mater. Chem. 2016, 4, 1486-1494.

(8) Wakim, S.; Beaupre, S.; Blouin, N.; Aich, B.-R.; Rodman, S.; Gaudiana, R.; Tao, Y.; Leclerc, M., Highly efficient organic solar cells based on a poly(2,7-carbazole) derivative. J. Mater. Chem. 2009, 19, 5351-5358.

(9) Sun, Y.; Welch, G. C.; Leong, W. L.; Takacs, C. J.; Bazan, G. C.; Heeger, A. J., Solution-processed small-molecule solar cells with $6.7 \%$ efficiency. Nat. Mater. 201 1, 11, 44-48.
(10) Zhou, H.; Zhang, Y.; Mai, C.-K.; Seifter, J.; Nguyen, T.-Q.; Bazan, G. C.; Heeger, A. J., Solution-Processed pH-Neutral Conjugated Polyelectrolyte Improves Interfacial Contact in Organic Solar Cells. ACS Nano 2015, 9, 371-377.

(11) Kyaw, A. K.; Wang, D. H.; Gupta, V.; Zhang, J.; Chand, S.; Bazan, G. C.; Heeger, A. J., Efficient solution-processed small-molecule solar cells with inverted structure. Adv. Mater. 2013, 25, 2397-2402.

(12) Wolf, J.; Babics, M.; Wang, K.; Saleem, Q.; Liang, R.-Z.; Hansen, M. R.; Beaujuge, P. M., Benzo[1,2-b:4,5- $\left.\boldsymbol{b}^{\prime}\right]$ dithiophene-Pyrido[3,4$b]$ pyrazine Small-Molecule Donors for Bulk Heterojunction Solar Cells. Chem. Mater. 2016, 28, 2058-2066.

(13) Kwon, O. K.; Park, J. H.; Kim, D. W.; Park, S. K.; Park, S. Y., An all-small-molecule organic solar cell with high efficiency nonfullerene acceptor. Adv. Mater. 2015, 27, 1951-1956.

(14) Wang, K.; Azouz, M.; Babics, M.; Cruciani, F.; Marszalek, T.; Saleem, Q.; Pisula, W.; Beaujuge, P. M., Solvent Annealing Effects in Dithieno $\left[3,2-b: 2^{\prime}, 3^{\prime}-d\right]$ pyrrole-5,6-

Difluorobenzo $[c][1,2,5]$ thiadiazole Small Molecule Donors for BulkHeterojunction Solar Cells. Chem. Mater. 2016, 28, 5415-5425.

(15) Wang, J.-L.; Liu, K.-K.; Yan, J.; Wu, Z.; Liu, F.; Xiao, F.; Chang, Z.-F.; Wu, H.-B.; Cao, Y.; Russell, T. P., Series of Multifluorine Substituted Oligomers for Organic Solar Cells with Efficiency over 9\% and Fill Factor of 0.77 by Combination Thermal and Solvent Vapor Annealing. J. Am. Chem. Soc. 2016, 138, 7687-7697.

(16) Sun, K.; Xiao, Z.; Hanssen, E.; Klein, M. F. G.; Dam, H. H.; Pfaff, M.; Gerthsen, D.; Wong, W. W. H.; Jones, D. J., The role of solvent vapor annealing in highly efficient air-processed small molecule solar cells. J. Mater. Chem. 2014, 2, 9048-9054.

(17) Sun, K.; Xiao, Z.; Lu, S.; Zajaczkowski, W.; Pisula, W.; Hanssen, E.; White, J. M.; Williamson, R. M.; Subbiah, J.; Ouyang, J.; Holmes, A. B.; Wong, W. W. H.; Jones, D. J., A molecular nematic liquid crystalline material for high-performance organic photovoltaics. Nat. Commun. 2015, 6, 6013.

(18) Mukherjee, S.; Proctor, C. M.; Tumbleston, J. R.; Bazan, G. C.; Nguyen, T.-Q.; Ade, H., Importance of Domain Purity and Molecular Packing in Efficient Solution-Processed Small-Molecule Solar Cells. Adv. Mater. 2015, 27, 1105-1111.

(19) Mukherjee, S.; Proctor, C. M.; Bazan, G. C.; Nguyen, T. Q.; Ade, H., Significance of Average Domain Purity and Mixed Domains on the Photovoltaic Performance of High-Efficiency Solution-Processed Small-Molecule BHJ Solar Cells. Adv. Energy Mater. 2015, 5, 1500877. (20) Yan, Y.; Liu, X.; Wang, T., Conjugated-Polymer Blends for Organic Photovoltaics: Rational Control of Vertical Stratification for High Performance. Adv. Mater. 2017, 29, 1601674.

(21) Ben Dkhil, S.; Pfannmöller, M.; Ata, I.; Duché, D.; Gaceur, M.; Koganezawa, T.; Yoshimoto, N.; Simon, J.-J.; Escoubas, L.; VidelotAckermann, C.; Margeat, O.; Bals, S.; Bäuerle, P.; Ackermann, J., Time evolution studies of dithieno[3,2-b:2',3'-d]pyrrole-based A-D-A oligothiophene bulk heterojunctions during solvent vapor annealing towards optimization of photocurrent generation. J. Mater. Chem. A 2017, 5, 1005-1013.

(22) Engmann, S.; Ro, H. W.; Herzing, A.; Snyder, C. R.; Richter, L. J.; Geraghty, P. B.; Jones, D. J., Film morphology evolution during solvent vapor annealing of highly efficient small molecule donor/acceptor blends. J. Mater. Chem. 2016, 4, 15511-15521.

(23) Min, J.; Jiao, X. C.; Ata, I.; Osvet, A.; Ameri, T.; Bauerle, P.; Ade, H.; Brabec, C. J., Time-Dependent Morphology Evolution of SolutionProcessed Small Molecule Solar Cells during Solvent Vapor Annealing. Adv. Energy Mater. 2016, 6, 1502579.

(24) Liang, R.-Z.; Wang, K.; Wolf, J.; Babics, M.; Wucher, P.; Thehaiban, M. K. A.; Beaujuge, P. M., Benzo[1,2-b:4,5- 
$\boldsymbol{b}^{\prime}$ ]Dithiophene-6,7-Difluoroquinoxaline Small Molecule Donors with $>8 \%$ BHJ Solar Cell Efficiency. Adv. Energy Mater. 2017, 1602804.

(25) Oldham, W. J.; Lachicotte, R. J.; Bazan, G. C., Synthesis, Spectroscopy, and Morphology of Tetrastilbenoidmethanes. J. Am. Chem. Soc. 1998, 120, 2987-2988.

(26) Halkyard, C. E.; Rampey, M. E.; Kloppenburg, L.; StuderMartinez, S. L.; Bunz, U. H. F., Evidence of Aggregate Formation for 2,5-Dialkylpoly( $p$-phenyleneethynylenes) in Solution and Thin Films. Macromolecules 1998, 31, 8655-8659.

(27) Lee, J.; Ko, H.; Song, E.; Kim, H. G.; Cho, K., Naphthodithiophene-Based Conjugated Polymer with Linear, Planar Backbone Conformation and Strong Intermolecular Packing for Efficient Organic Solar Cells. ACS Appl. Mater. Interfaces 2015, 7, 21159-21169.

(28) Spano, F. C.; Silva, C., H- and J-Aggregate Behavior in Polymeric Semiconductors. Annu. Rev. Phys. Chem. 2014, 65, 477-500.

(29) Schulz, G. L.; Ludwigs, S., Controlled Crystallization of Conjugated Polymer Films from Solution and Solvent Vapor for Polymer Electronics. Adv. Funct. Mater. 2017, 27, 1603083.

(30) Panzer, F.; Bassler, H.; Kohler, A., Temperature Induced OrderDisorder Transition in Solutions of Conjugated Polymers Probed by Optical Spectroscopy. J. Phys. Chem. Lett. 2017, 8, 114-125.

(31) Müller, C.; Ferenczi, T. A. M.; Campoy-Quiles, M.; Frost, J. M.; Bradley, D. D. C.; Smith, P.; Stingelin-Stutzmann, N.; Nelson, J., Binary Organic Photovoltaic Blends: A Simple Rationale for Optimum Compositions. Adv. Mater. 2008, 20, 3510-3515.

(32) Müller, C., On the Glass Transition of Polymer Semiconductors and Its Impact on Polymer Solar Cell Stability. Chem. Mater. 2015, 27, 2740-2754.

(33) Westacott, P.; Treat, N. D.; Martin, J.; Bannock, J. H.; de Mello, J. C.; Chabinyc, M.; Sieval, A. B.; Michels, J. J.; Stingelin, N., Origin of fullerene-induced vitrification of fullerene:donor polymer photovoltaic blends and its impact on solar cell performance. J. Mater. Chem. 2017, 5, 2689-2700.

(34) Min, J.; Luponosov, Y. N.; Gasparini, N.; Richter, M.; Bakirov, A. V.; Shcherbina, M. A.; Chvalun, S. N.; Grodd, L.; Grigorian, S.; Ameri, T.; Ponomarenko, S. A.; Brabec, C. J., Effects of Alkyl Terminal Chains on Morphology, Charge Generation, Transport, and Recombination Mechanisms in Solution-Processed Small Molecule Bulk Heterojunction Solar Cells. Adv. Energy Mater. 2015, 1500386.

(35) Wolf, J.; Cruciani, F.; El Labban, A.; Beaujuge, P. M., Wide BandGap 3,4-Difluorothiophene-Based Polymer with 7\% Solar Cell Efficiency: An Alternative to P3HT. Chem. Mater. 2015, 27, 41844187.

(36) Koster, L. J. A.; Kemerink, M.; Wienk, M. M.; Maturová, K.; Janssen, R. A. J., Quantifying Bimolecular Recombination Losses in Organic Bulk Heterojunction Solar Cells. Adv. Mater. 2011, 23, 16701674.

(37) Cowan, S. R.; Roy, A.; Heeger, A. J., Recombination in polymerfullerene bulk heterojunction solar cells. Phys. Rev. B: Condens. Matter 2010, 82, 245207.

(38) Armin, A.; Juska, G.; Ullah, M.; Velusamy, M.; Burn, P. L.; Meredith, P.; Pivrikas, A., Balanced Carrier Mobilities: Not a Necessary Condition for High-Efficiency Thin Organic Solar Cells as Determined by MIS-CELIV. Adv. Energy Mater. 2014, 4, 1300954.

(39) Proctor, C. M.; Love, J. A.; Nguyen, T. Q., Mobility guidelines for high fill factor solution-processed small molecule solar cells. Adv. Mater. 2014, 26, 5957-5961.

(40) Pearson, A. J.; Hopkinson, P. E.; Couderc, E.; Domanski, K.; Abdi-Jalebi, M.; Greenham, N. C., Critical light instability in CB/DIO processed PBDTTT-EFT:PC ${ }_{71} \mathrm{BM}$ organic photovoltaic devices. Org. Electron. 2016, 30, 225-236.

(41) Li, Z.; McNeill, C. R., Transient photocurrent measurements of PCDTBT:PC70BM and PCPDTBT:PC70BM Solar Cells: Evidence for charge trapping in efficient polymer/fullerene blends. J. Appl. Phys. 2011, 109, 074513.

(42) McNeill, C. R.; Hwang, I.; Greenham, N. C., Photocurrent transients in all-polymer solar cells: Trapping and detrapping effects. J. Appl. Phys. 2009, 106, 024507.

(43) Gao, F.; Li, Z.; Wang, J.; Rao, A.; Howard, I. A.; Abrusci, A.; Massip, S.; McNeill, C. R.; Greenham, N. C., Trap-Induced Losses in Hybrid Photovoltaics. ACS Nano 2014, 8, 3213-3221.

(44) Li, Z.; Gao, F.; Greenham, N. C.; McNeill, C. R., Comparison of the Operation of Polymer/Fullerene, Polymer/Polymer, and Polymer/Nanocrystal Solar Cells: A Transient Photocurrent and Photovoltage Study. Adv. Funct. Mater. 2011 , 21, 1419-1431.

(45) Shuttle, C. G.; Hamilton, R.; Nelson, J.; O'Regan, B. C.; Durrant, J. R., Measurement of Charge-Density Dependence of Carrier Mobility in an Organic Semiconductor Blend. Adv. Funct. Mater. 2010, 20, 698702 .

(46) Hwang, I.; McNeill, C. R.; Greenham, N. C., Drift-diffusion modeling of photocurrent transients in bulk heterojunction solar cells. J. Appl. Phys. 2009, 106, 094506.

(47) Tremolet de Villers, B. J.; MacKenzie, R. C. I.; Jasieniak, J. J.; Treat, N. D.; Chabinyc, M. L., Linking Vertical Bulk-Heterojunction Composition and Transient Photocurrent Dynamics in Organic Solar Cells with Solution-Processed MoOx Contact Layers. Adv. Energy Mater. 2014, 4, 1301290.

(48) Kniepert, J.; Schubert, M.; Blakesley, J. C.; Neher, D., Photogeneration and Recombination in P3HT/PCBM Solar Cells Probed by Time-Delayed Collection Field Experiments. J. Phys. Chem. Lett. 2011, 2, 700-705.

(49) Kniepert, J.; Lange, I.; Kaap, N. J. v. d.; Koster, L. J. A.; Neher, D., A Conclusive View on Charge Generation, Recombination, and Extraction in As-Prepared and Annealed P3HT:PCBM Blends: Combined Experimental and Simulation Work. Adv. Energy Mater. 2014, 4, 1301401.

(50) Albrecht, S.; Schindler, W.; Kurpiers, J.; Kniepert, J.; Blakesley, J. C.; Dumsch, I.; Allard, S.; Fostiropoulos, K.; Scherf, U.; Neher, D., On the Field Dependence of Free Charge Carrier Generation and Recombination in Blends of PCPDTBT/ $\mathrm{PC}_{70} \mathrm{BM}$ : Influence of Solvent Additives. J. Phys. Chem. Lett. 2012, 3, 640-645.

(51) Albrecht, S.; Janietz, S.; Schindler, W.; Frisch, J.; Kurpiers, J.; Kniepert, J.; Inal, S.; Pingel, P.; Fostiropoulos, K.; Koch, N.; Neher, D., Fluorinated Copolymer PCPDTBT with Enhanced Open-Circuit Voltage and Reduced Recombination for Highly Efficient Polymer Solar Cells. J. Am. Chem. Soc. 2012, 134, 14932-14944.

(52) Foertig, A.; Kniepert, J.; Gluecker, M.; Brenner, T.; Dyakonov, V.; Neher, D.; Deibel, C., Nongeminate and Geminate Recombination in PTB7:PCBM Solar Cells. Adv. Funct. Mater. 2014, 24, 1306-1311.

(53) Proctor, C. M.; Albrecht, S.; Kuik, M.; Neher, D.; Nguyen, T.-Q., Overcoming Geminate Recombination and Enhancing Extraction in Solution-Processed Small Molecule Solar Cells. Adv. Energy Mater. 2014, 4, 1400230.

(54) Burke, T. M.; McGehee, M. D., How High Local Charge Carrier Mobility and an Energy Cascade in a Three-Phase Bulk Heterojunction Enable $>90 \%$ Quantum Efficiency. Adv. Mater. 2014, 26, 1923-1928.

(55) Groves, C., Suppression of geminate charge recombination in organic photovoltaic devices with a cascaded energy heterojunction. Energy Environ. Sci. 2013, 6, 1546-1551. 


\title{
Solvent Vapor Annealing-Mediated Crystallization Directs Charge Generation, Recombination and Extraction in BHJ Solar Cells
}

\author{
Maxime Babics, ${ }^{\dagger}$ Ru-Ze Liang, ${ }^{\dagger}$ Kai Wang, ${ }^{\dagger}$ Federico Cruciani, ${ }^{+}$Zhipeng Kan, ${ }^{+}$Markus Wohlfahrt, ${ }^{+}$Ming-Chun \\ Tang, ${ }^{\dagger}$ Frederic Laquai, ${ }^{+}$and Pierre M. Beaujuge ${ }^{*,+}$
}

\section{TOC Figure}

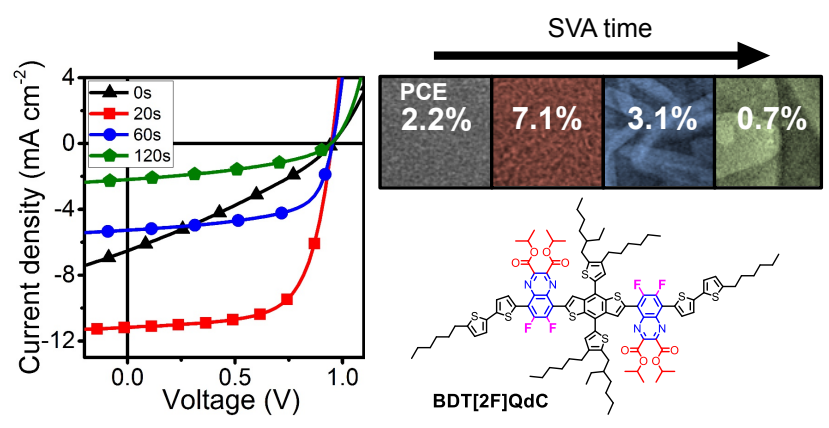

